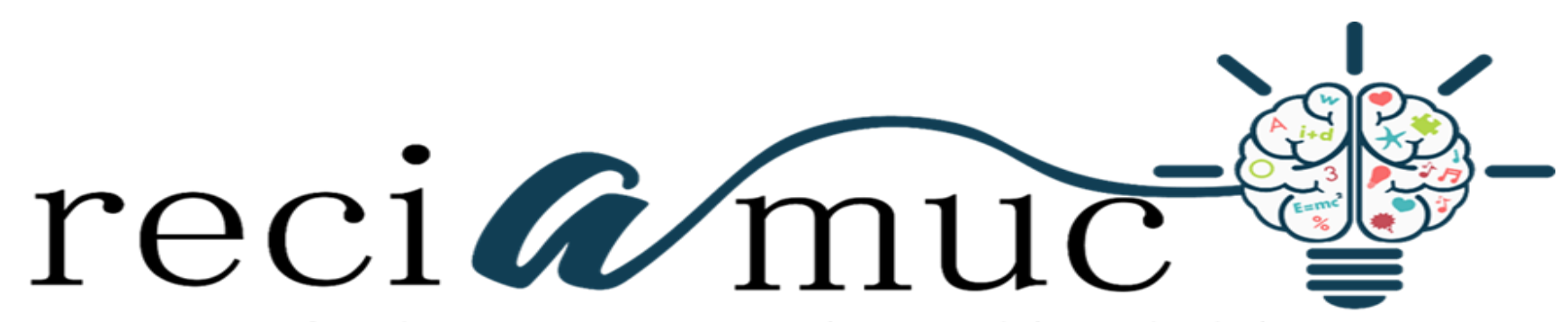

Revista cientifica de investígación actualización del mundo de las ciencias

Mery Adriana Franco Carrasco a ; Susana Del Carmen Yanez Carrasco ${ }^{\text {b; }}$; Marcela Sabrina Delgado Peña ${ }^{c}$; Stephanie Patrícia Nuñez Díaz ${ }^{d}$

El ejercicio combinado como prevención de la diabetes mellitus tipo II (DM2)

Combined exercise as prevention of type II diabetes mellitus (DM2)

Revista Científica de Investigación actualización del mundo de las Ciencias. Vol. 3 núm., 4, octubre: 2588-0748, 2019, pp. 123-142

DOI: 10.26820/reciamис/3.(4).octubre.2019.123-142

URL: http://reciamuc.com/index.php/RECIAMUC/article/view/400

Código UNESCO: 3205 Medicina Interna

Tipo de Investigación: Artículo de Revisión

(C) RECIAMUC; Editorial Saberes del Conocimiento, 2019

Recibido: $15 / 05 / 2019$

Aceptado: 07/08/2019

Publicado: 01/10/2019

Correspondencia: meryjean 21@ hotmail.com
a. Médico; Investigador Independiente; Guayaquil, Ecuador; meryjean_21@ @otmail.com
b. Médico; Investigador Independiente; Guayaquil, Ecuador; susy_medicina@ @otmail.com
c. Médico; Investigador Independiente; Guayaquil, Ecuador; marcelita_sama @hotmail.com
d. Médico; Investigador Independiente; Guayaquil, Ecuador; stephanie.nunezd@ gmail.com 


\section{El ejercicio combinado como prevención de la diabetes mellitus tipo II (DM2) \\ Vol. 3, núm. 4., (2019) \\ Mery Adriana Franco Carrasco; Susana Del Carmen Yanez Carrasco; Marcela Sabrina Delgado Peña; Stephanie Patrícia Nuñez Díaz}

\section{RESUMEN}

La diabetes está referida a una condición crónica que se origina a partir de que el páncreas no produce suficiente insulina o cuando la insulina que se produce no es utilizada eficazmente el organismo. La hormona de la insulina es la que regula el azúcar en la sangre. Cuando la diabetes no está bajo control, se deriva la hiperglucemia (aumento del azúcar en la sangre), que posteriormente puede dañar gravemente varios órganos y sistemas, principalmente los nervios y los vasos sanguíneos. Una de las formas de tratar la diabetes mellitus (DM) es mediante la realización del ejercicio físico, ya que éste se vuelve fundamental para el control glucémico. La efectividad de éste tipo de actividades físicas ha sido comprobada para éste tipo de complicaciones, como medio de prevención, sustancialmente en aquellos individuos influenciados por factores de alto riesgo, tales como: el sobrepeso, la tensión arterial elevada y con antecedentes genéticos de diabetes. Se ha desarrollado una revisión sistemática en el marco de una investigación documental secundaria, con la finalidad de constatar que el ejercicio aeróbico y de fuerza puede prevenir la aparición de la diabetes mellitus tipo 2 (DM2). Los resultados arrojaron contenido científico académico que efectivamente dieron satisfizo el objetivo propuesto y además facilitó el desarrollo de otros aspectos, igualmente necesarios e importantes para la comprensión de esta temática. En conclusión, se destaca la práctica de ejercicio físico combinado como una forma de tratamiento terapéutico de la DM2, siempre bajo la indicación y orientación de un especialista o profesional de la salud que pueda ajustar a cada individuo en particular. Igualmente se verifican las características mínimas que debe contener un plan de ejercitación y la adaptabilidad que éste debe tener, inclusive, respecto a las condiciones personales de edad, sexo y nivel socioeconómico.

Palabras claves: Crónica; Hormona; Físico; Complicaciones; Plan. 


\title{
El ejercicio combinado como prevención de la diabetes mellitus tipo II (DM2)
}

Vol. 3, núm. 4., (2019)

Mery Adriana Franco Carrasco; Susana Del Carmen Yanez Carrasco; Marcela Sabrina Delgado

Peña; Stephanie Patrícia Nuñez Díaz

\begin{abstract}
Diabetes is referred to a chronic condition that originates from the fact that the pancreas does not produce enough insulin or when the insulin that is produced is not used effectively by the body. Insulin hormone is what regulates blood sugar. When diabetes is not under control, hyperglycemia (increased blood sugar) is derived, which can subsequently severely damage several organs and systems, mainly nerves and blood vessels. One of the ways to treat diabetes mellitus (DM) is by performing physical exercise, since it becomes essential for glycemic control. The effectiveness of this type of physical activities has been proven for this type of complications, as a means of prevention, substantially in those individuals influenced by high risk factors, such as: overweight, high blood pressure and a genetic history of diabetes. A systematic review has been developed within the framework of a secondary documentary investigation, in order to confirm that aerobic and strength exercise can prevent the onset of type 2 diabetes mellitus (DM2). The results yielded scientific-academic content that actually gave met the proposed objective and also facilitated the development of other aspects, equally necessary and important for the understanding of this subject. In conclusion, the practice of combined physical exercise stands out as a form of therapeutic treatment of DM2, always under the direction and guidance of a specialist or health professional who can adjust to each individual in particular. Likewise, the minimum characteristics that an exercise plan must contain and the adaptability that it must have, including, regarding personal conditions of age, sex and socioeconomic status are verified.
\end{abstract}

Key words: Chronicle; Hormone; Physical; Complications; Plan. 


\section{El ejercicio combinado como prevención de la diabetes mellitus tipo II (DM2) \\ Vol. 3, núm. 4., (2019) \\ Mery Adriana Franco Carrasco; Susana Del Carmen Yanez Carrasco; Marcela Sabrina Delgado Peña; Stephanie Patrícia Nuñez Díaz}

\section{Introducción.}

Una de las formas de tratar la diabetes mellitus (DM) es mediante la realización del ejercicio físico, ya que éste se vuelve fundamental para el control glucémico. La efectividad de éste tipo de actividades físicas ha sido comprobada para éste tipo de complicaciones, como medio de prevención, sustancialmente en aquellos individuos influenciados por factores de alto riesgo, tales como: el sobrepeso, la tensión arterial elevada y con antecedentes genéticos de diabetes.

Evidentemente, la diabetes mellitus tipo II (DM2) no es la excepción para este tipo de medidas, así lo da a entender Murillo (2015), pues indica que el ejercicio influye directamente sobre los valores de glucosa en sangre, es decir, se logra un efecto mayor sobre las cifras de glucosa en sangre. De la misma destaca que, al respecto, recientemente se ha recomendado la combinación de ejercicios aeróbicos y de fuerza, que en términos sencillos se refieren a: caminar, ir en bicicleta o nadar; en conjunto de 2 a 3 sesiones de ejercicios usando pesas, máquinas o cintas elásticas, respectivamente. Ésta combinación ha demostrado su efectividad en el control de la diabetes, aunque también facilita la reducción del riesgo de padecimiento de problemas cardiovasculares y la mortalidad. "En definitiva, la práctica de ejercicio físico debe ser fundamental en las personas con diabetes, sea cual sea su edad o tipo de diabetes". (Murillo, 2015)

Los datos estadísticos de la Federación Internacional de Diabetes estiman que existe una alta prevalencia de 382 millones de adultos con esta enfermedad a nivel mundial, siendo que el número de personas con diabetes en el año 2035 será de aproximadamente 592 millones de individuos. (Cascaes et al.; 2017; p. 13) 


\section{El ejercicio combinado como prevención de la diabetes mellitus tipo II (DM2)}

Vol. 3, núm. 4., (2019)

Mery Adriana Franco Carrasco; Susana Del Carmen Yanez Carrasco; Marcela Sabrina Delgado Peña; Stephanie Patrícia Nuñez Díaz

En base a la información aportada por la Organización Mundial de la Salud (OMS), la diabetes está referida a una condición crónica que se origina a partir de que el páncreas no produce suficiente insulina o cuando la insulina que se produce no es utilizada eficazmente el organismo. Al mismo tiempo explica que la hormona de la insulina es la que regula el azúcar en la sangre. Cuando la diabetes no está bajo control, se deriva la hiperglucemia (aumento del azúcar en la sangre), que posteriormente puede dañar gravemente varios órganos y sistemas, principalmente los nervios y los vasos sanguíneos. "Aproximadamente, la mitad de las muertes atribuibles a la hiperglucemia tienen lugar antes de los 70 años de edad." Este organismo proyecta que para el año 2030, la diabetes será la séptima causa de mortalidad a nivel mundial. "La dieta saludable, la actividad física regular, el mantenimiento de un peso corporal normal y la evitación del consumo de tabaco previenen la diabetes de tipo 2 o retrasan su aparición". (OMS, 2018)

En concreto, se desarrollará una exposición enfocada en referir los criterios medicocientíficos que fundamentan la premisa de la prevención de la DM2 mediante la actividad de ejercicios físicos combinados, e igualmente, se destacarán otros aspectos necesarios e importantes para la comprensión de esta temática.

\section{Materiales y Métodos.}

En el marco de una investigación documental secundaria, se ha desarrollado una revisión sistemática, con la finalidad de recurrir a la búsqueda y utilización de diversos materiales y fuentes bibliográficas formales, accedida por medio del uso de diversas bases de datos, tales como: OMS, PubMed, Redalyc, Imbiomed, SciELO, Dialnet, MedlinePlus, OPS/PAHO, Intramed, EBSCO, ScienceDirect, LILACS, Embase, entre otras. 


\section{El ejercicio combinado como prevención de la diabetes mellitus tipo II (DM2)}

Vol. 3, núm. 4., (2019)

Mery Adriana Franco Carrasco; Susana Del Carmen Yanez Carrasco; Marcela Sabrina Delgado Peña; Stephanie Patrícia Nuñez Díaz

En función de constatar que el ejercicio aeróbico y de fuerza puede prevenir la aparición de la DM2, inicialmente se buscó contenido cientificoacadémico clasificado como: artículos científicos de revistas online o digitalizadas, guías de manejo o de práctica clínica, revisiones sistemáticas con o sin metaanálisis, ensayos clínicos, boletines y/o folletos informativos de organismos oficiales de carácter nacional e internacional, tesis de postgrado o doctorado, datos estadísticos, entre otros tipos de documentos.

Seguidamente, tras la obtención de los resultados por las múltiples búsquedas realizadas en las distintas bases de datos antes mencionadas, se efectuó el ordenamiento, clasificación y selección de los títulos que, a criterio del equipo de trabajo, se consideró más relevante, en base a parámetros selectivos preestablecidos, tales como: contenido en idioma español (preferiblemente) e inglés en menor proporción; mayor correlación temática en la medida de lo posible; publicación hecha en los últimos 10 años, salvo algunas excepciones, y; desestimación de fuentes bibliográficas alusivas a estudios de cohorte, casos y controles; series y reportes de casos, editoriales y cartas al editor (Editoriales). Este proceso resultó en la escogencia de más de veinticinco títulos que, como fuentes de información, permitieron fundamentar las bases teóricas y científicas de la argumentación aquí plasmada, en definitiva, facilitando la construcción del criterio conjunto que se ha procurado dar a entender entre las partes que componen el presente desarrollo investigativo.

\section{Resultados.}

Un factor clave en el desarrollo de la diabetes es la resistencia a la insulina, asegura Murillo (2011). Es por ello que su reducción se puede logar practicando regularmente algún tipo de 


\section{El ejercicio combinado como prevención de la diabetes mellitus tipo II (DM2)}

Vol. 3, núm. 4., (2019)

Mery Adriana Franco Carrasco; Susana Del Carmen Yanez Carrasco; Marcela Sabrina Delgado Peña; Stephanie Patrícia Nuñez Díaz

ejercicio físico, ya que además de mejorar dicho factor, aporta otros beneficios importantes tales como la mejora de la presión arterial, los niveles de colesterol y ayuda a controlar el peso corporal, sin menoscabo de los beneficios que a nivel psicológico también se consigues, "como la disminución de los niveles de ansiedad o el aumento de la autoestima y la autoconfianza".

Desde hace años muchos estudios demuestran que aquellas personas que realizan ejercicio físico de forma habitual desarrollan menos casos de diabetes tipo 2 que aquellas otras que permanecen sedentarias, sobre todo en aquellas personas que ya tienen alto riesgo de padecer diabetes. (Murillo, 2011)

Sesmilo \& Pérez (2017) indican que alternar entre ejercicios físicos aeróbicos y de fuerza, que en la semana sumen más de 2,5 horas, "puede ayudar a mejorar el control metabólico de las personas con diabetes tipo 2". Igualmente coinciden con el criterio de los beneficios físicos y mentales que aporta la ejecución regular de cualquier actividad física, enfatizando además la gran herramienta terapéutica que representan este tipo de actividades en pacientes con DM2, por cuanto: mejora los niveles de glucosa en sangre, aumenta la sensibilidad a la insulina, disminuye los lípidos en sangre, mejora la presión arterial, ayuda a reducir la grasa corporal y bajar de peso, disminuye el riesgo de padecer enfermedades cardiovasculares, reduce la ansiedad, aumenta la autoestima y hace que nos sintamos y nos veamos mejor, y resulta ser terapéutico, no solo para el estado de ánimo sino también para la memoria.

Estas expertas alegan que se ha evidenciado mediante estudios recientes que el ejercicio físico hecho a corto y largo plazo hace posible un mejor control glucémico en la DM2, ya que los 


\section{El ejercicio combinado como prevención de la diabetes mellitus tipo II (DM2)}

Vol. 3, núm. 4., (2019)

Mery Adriana Franco Carrasco; Susana Del Carmen Yanez Carrasco; Marcela Sabrina Delgado Peña; Stephanie Patrícia Nuñez Díaz

niveles de glucosa en sangre y de la hemoglobina glicosilada (HbA1c -resumen de la glucosa de los últimos 3-4 meses-) son reducidas formidablemente.

De la misma manera han referido que en otro estudio en el que se buscaba "determinar los beneficios de cada tipo de ejercicio físico", se evaluó "el efecto del entrenamiento sobre el control glucémico a largo plazo (medido por la hemoglobina glicosilada), y sobre el control glucémico 24-48h post ejercicio con distintas modalidades de ejercicio (medido con valores de monitorización continua de glucosa). Respecto a la primera de las situaciones planteadas los resultados fueron favorables, sin embargo, más satisfactorios fueron éstos cuando se trataba de programas de ejercicio físico estructurados durante más de 12 semanas. En relación al segundo planteamiento, en el estudio se observó (tras una sesión de entrenamiento): disminución del tiempo en hiperglucemia, disminución de la glucosa post ejercicio físico, disminución de glucosa hasta 48h post ejercicio físico.

Así mismo, estas investigadoras dan a entender que con dicho estudio también se evidenció que "con ejercicio físico aeróbico (cardiovascular), con ejercicio físico de fuerza y con combinación de ambos, reducciones significativas de 0,5-0,8\% en la HbA1c. Sin embargo, las mayores reducciones son las asociadas a la combinación de ejercicio cardiovascular y de fuerza."

\section{Influencia del ejercicio en el control de la glucosa en la sangre}

La Asociación Americana de Diabetes (ADA, por sus siglas en inglés) ha indicado que la glucosa en la sangre puede ser disminuida a corto plazo mediante la realización del ejercicio físico de varias maneras, entre las que destacan: 


\section{El ejercicio combinado como prevención de la diabetes mellitus tipo II (DM2)}

Vol. 3, núm. 4., (2019)

Mery Adriana Franco Carrasco; Susana Del Carmen Yanez Carrasco; Marcela Sabrina Delgado Peña; Stephanie Patrícia Nuñez Díaz

- Se aumenta la sensibilidad a la insulina, por lo que las células pueden aprovechar más cualquier insulina disponible para usar glucosa mientras hace actividad física y después.

- Cuando los músculos se contraen durante la actividad, se estimula otro mecanismo totalmente separado de la insulina. Este mecanismo permite que las células tomen glucosa y la utilicen como fuente de energía, independientemente de si hay insulina disponible.

Además, refieren que, de realizarse de manera regular la actividad física, también es posible que se logre disminuir el porcentaje de la prueba de glucosa promedio estimada (A1C). (ADA, 2013 a)

Beneficios del ejercicio físico en DM2

Bazán (2014) explica que definitivamente, un elemento primordial en el proceso de conseguir el equilibrio calórico es la ejecución de una actividad física, más aún en personas diabéticas, ya que en el tratamiento representa un aspecto tan trascendental como la nutrición o la medicación, de allí su papel clave en la fase preventiva.

Asegura que el ejercicio diario, o sea, regular, mejora el control de la glucemia, y coadyuva en la disminución de las dosis de medicación, disminuye los valores de hemoglobina glicosilada, siendo éste positivo impacto proporcional a los niveles más altos de la intensidad del ejercicio, inclusive, sin ningún cambio significativo en el Índice de Masa Corporal (IMC). Además, favorece el control de peso y disminución de masa adiposa. Los otros beneficios podrían ser: 


\section{El ejercicio combinado como prevención de la diabetes mellitus tipo II (DM2)}

Vol. 3, núm. 4., (2019)

Mery Adriana Franco Carrasco; Susana Del Carmen Yanez Carrasco; Marcela Sabrina Delgado Peña; Stephanie Patrícia Nuñez Díaz

- $\quad$ Disminuye de la glucemia.

- $\quad$ Mejora de la sensibilidad a la insulina por 12 a 72 horas.

- Disminución de los valores de tensión

- $\quad$ Mejora en el perfil lipídico: triglicéridos y colesterol arterial

- $\quad$ Mejora en la función cardiovascular, ya que promueve una menor frecuencia cardiaca en

- reposo, aumento del volumen latido y disminución del trabajo del corazón,

- $\quad$ Aumento de la fuerza y flexibilidad

- $\quad$ Mejora de la sensación de bienestar

- $\quad$ Mejora de la calidad de vida.

- $\quad$ Mejora en el control metabólico a largo plazo.

Durante el ejercicio físico el músculo utiliza su propia reserva de energía, que se encuentra almacenada como glucógeno. Al cabo de unos minutos comienza a utilizarse la glucosa circulante en la sangre y es en esta fase cuando la glucemia tiende a disminuir. Esto se debe a que el ejercicio promueve la aparición en membrana de los receptores musculares a glucosa, Glut4. Las grasas se utilizan de modo importante en las actividades continuas con intensidades cercanas al $60 \%$ de la frecuencia cardíaca de ejercitación. También se utilizarán grasas en los momentos de recuperación de ejercicios intermitentes. (Bazán, 2014)

\section{El ejercicio ideal}

La Fundación Española del Corazón (FEC) expone, sin distinción entre diabetes tipo 1 y 2, que: 


\section{El ejercicio combinado como prevención de la diabetes mellitus tipo II (DM2)}

Vol. 3, núm. 4., (2019)

Mery Adriana Franco Carrasco; Susana Del Carmen Yanez Carrasco; Marcela Sabrina Delgado

Peña; Stephanie Patrícia Nuñez Díaz

Una sesión debería constar de unos 10 a 20 minutos de estiramiento y de fuerza muscular, 5 minutos de calentamiento aeróbico (carrera suave), 15 a 60 minutos de ejercicio aeróbico a una intensidad apropiada y de 5 a 10 minutos de ejercicio de baja intensidad al acabar la práctica deportiva. Los deportes más recomendables son caminar, correr y montar en bicicleta, pero siempre hay que tener en cuenta el historial médico del paciente. Los diabéticos deben llevar un registro de entrenamiento y consultar siempre con su médico antes de realizar un ejercicio de forma continuada. (FEC, 2019)

\section{Medidas preventivas para la ejercitación de personas con DM2}

De acuerdo con la Federación Mexicana de Diabetes (FMD), se comprende que el ejercicio físico, de la mano con la alimentación y el tratamiento farmacológico, es considerado uno de los pilares fundamentales en el tratamiento de la DM2. Básicamente recomiendan realizar ejercicios un mínimo de 3 a 4 días por semana, no obstante, destacan que éstos deben estar adaptados a las propias necesidades del que la padece, por lo tanto, es imprescindible que cualquier programa de ejercicios sea planificado bajo control médico.

Precisan que la persona que recibe tratamiento con insulina, debe tener presente que la realización de una actividad física induce las alteraciones en los niveles de azúcar, por lo que evidentemente, deben efectuarse los ajustes adecuados en la dosis de insulina. Así mismo advierten que la idea es comenzar con actividades físicas de intensidad constante moderada o baja, en vista de que representan el mejor equilibrio entre los beneficios esperados y los riesgos en que se puede incurrir, no obstante, al momento de elegir el ejercicio físico más adecuado, el(los) tratante(s) consecuentemente valorará(n) si existen o no complicación(es) por la DM2. 


\section{El ejercicio combinado como prevención de la diabetes mellitus tipo II (DM2)}

Vol. 3, núm. 4., (2019)

Mery Adriana Franco Carrasco; Susana Del Carmen Yanez Carrasco; Marcela Sabrina Delgado Peña; Stephanie Patrícia Nuñez Díaz

"Entre los ejercicios más recomendables, se incluyen actividades de carácter aeróbico como caminar, ir en bicicleta, correr o nadar, realizadas entre tres y cinco veces por semana, durante 2060 minutos." (FMD, 2016)

Las previsiones que recomiendan tomar son:

Antes del ejercicio:

- Comprobar los niveles de glucemia y actuar en base a lo acordado con el(los) especialista(s) tratante(s).

- $\quad$ En caso de tener que administrase insulina antes de iniciar el ejercicio, es recomendable reducir dicha dosis o aumentar la ingesta de alimentos ricos en carbohidratos.

- Evitar las inyecciones de insulina en zonas que van a ser sometidas a movimientos durante la realización del(los) ejercicio(s).

Durante el ejercicio:

- Siempre llevar carbohidratos de rápida absorción tales como: terrones de azúcar, pastillas de glucosa, bebidas isotónicas, entre otras, que puedan ser ingeridas velozmente en caso de aparición de síntomas de hipoglucemia.

- Realizar ejercicio en compañía de alguien e informarle acerca de cómo actuar frente a una hipoglucemia grave.

- $\quad$ Beber agua adecuadamente.

Después de la actividad: 


\section{El ejercicio combinado como prevención de la diabetes mellitus tipo II (DM2)}

Vol. 3, núm. 4., (2019)

Mery Adriana Franco Carrasco; Susana Del Carmen Yanez Carrasco; Marcela Sabrina Delgado

Peña; Stephanie Patrícia Nuñez Díaz

- Comprobar la glucemia capilar.

- $\quad$ En el caso de ejercicios prolongados, disminuir la dosis de insulina posterior o aumenta la ingesta de alimentos ricos en carbohidratos. (FMD, 2016)

Qué tipo de ejercicio ayuda a prevenir la enfermedad

Nuevamente la ADA (Asociación Americana de Diabetes) resalta particularmente al ejercicio aeróbico y el ejercicio con pesas, como las actividades físicas especialmente importantes en el control de la diabetes.

Respecto al ejercicio aeróbico, afirma que favorece un mejor uso de la insulina en el cuerpo. De la misma manera, indica que "fortalece el corazón y los huesos, alivia el estrés, mejora la circulación y disminuye el riesgo de enfermedades del corazón al reducir la glucosa en la sangre y la presión arterial, además de mejorar el nivel de colesterol". Recomiendan hacer este tipo de ejercicios durante 30 minutos, bajo una intensiva que oscile entre lo "intensa moderada a vigorosa" en mínimo 5 días semanales o de 150 minutos en total en el mismo periodo de tiempo. También recomiendan evitar dejar de hacer los ejercicios durante dos días seguidos y realizar la actividad física, por lo menos, durante 3 días en la semana. Aclaran que " Intensidad moderada significa que está esforzándose bastante, al punto que puede hablar, pero no cantar durante la actividad. Intensidad vigorosa significa que no puede decir más de unas pocas palabras sin detenerse para recobrar el aliento durante la actividad"

En relación al entrenamiento con pesas (o ejercicios de resistencia), exponen que los mismos generan una mayor sensibilidad a la insulina que el cuerpo y favorecen la reducción de la 


\section{El ejercicio combinado como prevención de la diabetes mellitus tipo II (DM2)}

Vol. 3, núm. 4., (2019)

Mery Adriana Franco Carrasco; Susana Del Carmen Yanez Carrasco; Marcela Sabrina Delgado Peña; Stephanie Patrícia Nuñez Díaz

glucosa en la sangre. Particularmente facilitan el fortalecimiento de músculos y huesos persistente, lo que deriva en la reducción del riesgo de fracturas de hueso y osteoporosis. Precisan que, mientras más se vaya desarrollando la masa muscular, mayor será la cantidad de calorías quemadas, incluso cuando con el cuerpo en estado de reposo. El ejercicio con pesas evita la pérdida de músculo. Recomiendan cualquier ejercicio con pesas de 2 a 3 días a la semanada, independientemente de la ejercitación aeróbica. (ADA, 2013 b)

Por su parte, la Fundación Red de Grupos de Estudios de la Diabetes en Atención Primaria de la Salud de España, en un trabajo más reciente, recomiendan una estructura un tanto más clara respecto a las mencionadas categorías de ejercicios, a saber:

\section{Ejercicios aeróbicos}

- $\quad$ Tipo de ejercicio: cualquier tipo de actividad que use grandes grupos musculares durante largos períodos de tiempo, incluyendo caminar a ritmo rápido.

- Frecuencia: como mínimo tres veces a la semana, en días no consecutivos. El objetivo será cinco sesiones semanales.

- Intensidad: las sesiones de ejercicio deben realizarse como mínimo a intensidad moderada, correspondiente al 40-60\% del consumo máximo de oxígeno, lo que equivale al 55-70 \% de la frecuencia cardíaca máxima.

- Duración: 150 minutos semanales si se realiza ejercicio a intensidad moderada (por ejemplo, cinco sesiones semanales de 30 minutos) o 60 minutos semanales a intensidad alta (entre el 60 y el $84 \%$ del consumo máximo de oxígeno o entre el 70 y el $89 \%$ de 


\section{El ejercicio combinado como prevención de la diabetes mellitus tipo II (DM2)}

Vol. 3, núm. 4., (2019)

Mery Adriana Franco Carrasco; Susana Del Carmen Yanez Carrasco; Marcela Sabrina Delgado Peña; Stephanie Patrícia Nuñez Díaz

la frecuencia cardíaca máxima, o bien una combinación equivalente entre ambos tipos de intensidades).

- $\quad$ Ritmo de progresión: se debe ajustar el aumento de las cargas de entrenamiento con el objetivo de evitar lesiones.

Ejercicios de fuerza muscular

- $\quad$ Tipo de ejercicio: ejercicios en los que se utilizan máquinas de resistencia o pesos libres como mancuernas o pesas.

- Frecuencia: como mínimo dos veces a la semana, en días no consecutivos. El objetivo será tres sesiones semanales.

- Intensidad: las sesiones de ejercicio deben realizarse como mínimo a intensidad moderada, correspondiente al $50 \%$ de una repetición máxima (o peso con el que el paciente es capaz de realizar una sola repetición del ejercicio), o a intensidad alta (el 75-80\% de una repetición máxima).

- Duración: en cada sesión se incluirán 5-10 ejercicios que utilicen grandes grupos musculares del organismo, realizando unas 10-15 repeticiones de cada ejercicio. Para cada ejercicio se efectuará un mínimo de una serie, llegando como máximo a 3-4 series por ejercicio.

- $\quad$ Ritmo de progresión: se incrementará el peso de cada ejercicio hasta que se llegue a estar cercano a la fatiga al realizar solamente 8-10 repeticiones de cada ejercicio. (Fundación redGDPS, 2016) 


\section{El ejercicio combinado como prevención de la diabetes mellitus tipo II (DM2)}

Vol. 3, núm. 4., (2019)

Mery Adriana Franco Carrasco; Susana Del Carmen Yanez Carrasco; Marcela Sabrina Delgado Peña; Stephanie Patrícia Nuñez Díaz

Según Ibáñez (2014) “el entrenamiento de fuerza puede ser un medio muy útil para el tratamiento de la obesidad y de la diabetes tipo 2", y esto es en vista de que, el entrenamiento de fuerza ayuda a la persona obesa que está siguiendo una dieta para perder peso porque:

- $\quad$ Reduce sustancialmente la pérdida de músculo que se produce cuando una persona hace sólo dieta. Mantener la masa muscular corporal es esencial para un mejor transporte de la glucosa y del metabolismo de la grasa.

- $\quad$ Evita la reducción del metabolismo de reposo que se produce inevitablemente cuando se sigue sólo una dieta hipocalórica.

- Ayuda a mejorar las cifras de la tensión arterial, del colesterol y de los triglicéridos.

- $\quad$ Mejora la fuerza, la flexibilidad y la resistencia física.

- $\quad$ Mejora el sentimiento de bienestar y autoestima.

- $\quad$ Es mucho más probable que aquellas personas que han perdido peso y han abandonado la dieta hipocalórica puedan mantener esta pérdida si siguen realizando el entrenamiento de fuerza. (Ibañez, 2014)

Ahora bien, Suárez (2019), como especialista aclara que, en el caso de los niños, aunque no existe ninguna contravención para que un niño diabético pueden realizar cualquier tipo de ejercicio, es importante tener claro que, entre los distintos tipos de ejercicios, está implícito su duración e intensidad, por lo que igualmente ellos pueden experimentar diferentes efectos en los niveles de glucosa en sangre. Igualmente menciona los ejercicios aeróbicos, que generan prácticamente los mismos efectos que en los adultos, haciendo la salvedad solo respectos a la hipoglucemia, que puede ocurrir "tanto durante la realización del ejercicio (entre 30 - 60 minutos 


\section{El ejercicio combinado como prevención de la diabetes mellitus tipo II (DM2)}

Vol. 3, núm. 4., (2019)

Mery Adriana Franco Carrasco; Susana Del Carmen Yanez Carrasco; Marcela Sabrina Delgado Peña; Stephanie Patrícia Nuñez Díaz

después del inicio) hasta 12 - 24 horas después de haberlo realizado". Respecto a los ejercicios de fuerza muscular, hace referencia a los ejercicios anaeróbicos, tales como: " la realización de abdominales y flexiones, los sprint (carreras cortas a gran velocidad) y levantamiento de pesas. Así mismo, deportes como el judo o la gimnasia" (Suárez, 2019)

Tabla 1. Adaptación de ejercicios en caso de complicaciones asociadas a diabetes

\begin{tabular}{|c|c|c|c|c|c|}
\hline & $\begin{array}{c}\text { Enfermedad } \\
\text { Cardiovascul } \\
\text { ar (Valorar } \\
\text { Prueba de } \\
\text { Esfuerzo) } \\
\end{array}$ & $\begin{array}{c}\text { Retinopatí } \\
\mathbf{a}\end{array}$ & $\begin{array}{c}\text { Nefropat } \\
\text { ía }\end{array}$ & $\begin{array}{l}\text { Neuropat } \\
\text { ía } \\
\text { Periférica }\end{array}$ & $\begin{array}{l}\text { Neuropatí } \\
\text { a } \\
\text { Autonómi } \\
\text { ca }\end{array}$ \\
\hline $\begin{array}{l}\text { Ejercicio } \\
\text { Recomendad } \\
\text { o }\end{array}$ & $\begin{array}{l}\text { Natación, } \\
\text { caminar, cinta } \\
\text { rodante, bicicleta } \\
\text { Estática }\end{array}$ & $\begin{array}{l}\text { Natación, } \\
\text { caminar, cinta } \\
\text { rodante, } \\
\text { bicicleta } \\
\text { Estática }\end{array}$ & $\begin{array}{l}\text { Natación, } \\
\text { caminar, } \\
\text { cinta } \\
\text { rodante, } \\
\text { bicicleta } \\
\text { Estática }\end{array}$ & $\begin{array}{l}\text { Natación, } \\
\text { bicicleta, } \\
\text { ejercicios de } \\
\text { silla, brazos } \\
\text { y } \\
\text { que no } \\
\text { requieran } \\
\text { utilizar los } \\
\text { pies }\end{array}$ & $\begin{array}{l}\text { Acuáticos, } \\
\text { bicicleta } \\
\text { estática, } \\
\text { ejercicios } \\
\text { sentado, } \\
\text { ciclismo < } 10 \\
\mathrm{~km} / \mathrm{h} o r a\end{array}$ \\
\hline Evitar & $\begin{array}{l}\text { Deportes que } \uparrow \\
\text { PA*si IAM }<6 \\
\text { semanas }\end{array}$ & $\begin{array}{l}\text { Si RD } \\
\text { proliferativa } \\
\text { activa y tras } \\
\text { fotocoagulació } \\
\text { n o cirugía } \\
\text { recientes. } \\
\text { Deportes que } \uparrow \\
\text { PA*, bajar la }^{*} \\
\text { cabeza, saltos } \\
\text { o } \\
\text { deportes de } \\
\text { contacto }\end{array}$ & $\begin{array}{l}\text { Deportes } \\
\text { que } \uparrow \mathrm{PA}^{*}\end{array}$ & $\begin{array}{l}\text { Caminatas } \\
\text { prolongadas, } \\
\text { correr, cinta, } \\
\text { saltar. } \\
\text { Ejercicio si } \\
\text { úlceras o pie } \\
\text { de Charcot } \\
\text { activo. } \\
\text { Ejercicio en } \\
\text { temperaturas } \\
\text { extremas }\end{array}$ & $\begin{array}{l}\text { Ejercicio de } \\
\text { elevada } \\
\text { intensidad, o } \\
\text { deportes que } \\
\uparrow \mathrm{PA}^{*}\end{array}$ \\
\hline
\end{tabular}

Nota: Tomada de: Grupo de Diabetes - Sociedad Española de Endocrinología y Nutrición [SEEN] (2018) 


\section{El ejercicio combinado como prevención de la diabetes mellitus tipo II (DM2)}

Vol. 3, núm. 4., (2019)

Mery Adriana Franco Carrasco; Susana Del Carmen Yanez Carrasco; Marcela Sabrina Delgado Peña; Stephanie Patrícia Nuñez Díaz

FC: frecuencia cardiaca; ECV: enfermedad cardiovascular; PA: presión arterial; IAM: infarto agudo de miocardio; RD: retinopatía.

*Actividades físicas violentas, movimientos que impliquen realización de Valsalva, ejercicios de contracción isométricos, levantamiento de pesas, boxeo, artes marciales.

\section{Conclusión}

Teniendo como fundamente la referencia científica aquí mencionada, primero que nada, debe destacarse que la práctica de ejercicio físico combinada para el tratamiento terapéutico de la DM2 debe ser, preferiblemente, indicada y orientada por un especialista o profesional de la salud acreditado, particularmente en cada individuo que padece esta condición, al margen de las limitaciones propias de cada paciente, y teniendo en perspectiva que, en términos generales, lo ideal vendría siendo ejecutar una combinación de ejercicios físicos (cardiovascular/aeróbico y de fuerza).

Como mínimo, la ejercitación combinada debe desarrollarse de manera alternada (sin dejar pasar más 48 horas entre ambos tipos), con una carga mínima semanal de150 minutos, en alta intensidad, al punto de lo que comúnmente se entiende como "perdiendo el habla", ya que, en esos momentos en los que, haciendo ejercicio cuesta hablar, precisamente es cuando se ha empezado a usar la glucosa del sistema corporal para reponer la energía gastada, correspondiendo esta intensidad, generalmente, con el 50 al 70\% de la frecuencia cardíaca máxima.

La prescripción de ejercicio físico no es un fenómeno de todo o nada. Deben valorarse positivamente incrementos moderados de la actividad física, puesto que está demostrado su efecto 


\section{El ejercicio combinado como prevención de la diabetes mellitus tipo II (DM2)}

Vol. 3, núm. 4., (2019)

Mery Adriana Franco Carrasco; Susana Del Carmen Yanez Carrasco; Marcela Sabrina Delgado Peña; Stephanie Patrícia Nuñez Díaz

beneficioso sobre la salud. Debe individualizarse y adaptarse a las condiciones personales de edad, sexo y nivel socioeconómico. (Fundación redGDPS, 2018)

\section{Bibliografía.}

ADA. (22 de Agosto de 2013 a). American Diabetes Association. Recuperado el 06 de 08 de 2019, de diabetes.org: http://archives.diabetes.org/es/alimentos-y-actividad-fisica/condicionfisica/empezar-de-forma-segura/el-ejercicio-y-el-control-de.html

ADA. (30 de Abril de 2013 b). American Diabetes Association. Recuperado el 10 de 08 de 2019, de diabetes.org: http://archives.diabetes.org/es/alimentos-y-actividad-fisica/condicionfisica/tipos-de-ejercicio/que-recomendamos.html

Bazán, N. (Junio de 2014). Actividad física en personas con diabetes. ISDe Sports Magazine. , 6(21), 7 p. Recuperado el 07 de 08 de 2019, de http://www.isde.com.ar/ojs/index.php/isdesportsmagazine/article/viewFile/115/133

Cascaes, F., Da Rosa, R., Valdivia, B., Barbosa, P., Da Silva, R., Olivera, M., \& Pereira, A. (2017). Ejercicio físico, calidad de vida y salud de diabéticos tipo 2. Revista de Psicología del Deporte, 26(1), 13-25. Recuperado el 12 de 08 de 2019, de https://core.ac.uk/download/pdf/78547106.pdf

FEC. (2019). Fundacion Española del Corazon. Recuperado el 09 de 08 de 2019, de https://fundaciondelcorazon.com: enfermos/980-diabetes-y-ejercicio.html https://fundaciondelcorazon.com/ejercicio/para-

FMD. (16 de Junio de 2016). Federación Mexicana de Diabetes, A.C. Recuperado el 10 de 08 de 2019, de fmdiabetes.org: http://fmdiabetes.org/ejercicio-y-diabetes-tipo-2/

Fundación redGDPS. (2016). Fundación redGDPS. Recuperado el 10 de 08 de 2019, de https://www.redgdps.org: https://www.redgdps.org/gestor/upload/GUIA2016/Guia_Actualizacion_2016v2.pdf

Fundación redGDPS. (2018). Fundación redGDPS. Recuperado el 12 de 08 de 2019, de redgdps.org: https://www.redgdps.org/gestor/upload/colecciones/Guia\%20DM2_web.pdf

Grupo de Diabetes - Sociedad Española de Endocrinología y Nutrición [SEEN]. (2018). Sociedad Española de Endocrinología y Nutrición. Recuperado el 10 de 08 de 2019, de seen.es: http://www.seen.es/docs/apartados/791/2018\%2005\%2005\%20Abordaje\%20Integral\%20 DM2_SEEN_2018_GTDMSEEN\%201.pdf 


\section{El ejercicio combinado como prevención de la diabetes mellitus tipo II (DM2)}

Vol. 3, núm. 4., (2019)

Mery Adriana Franco Carrasco; Susana Del Carmen Yanez Carrasco; Marcela Sabrina Delgado

Peña; Stephanie Patrícia Nuñez Díaz

Ibañez, J. (2014). Papel del entrenamiento de fuerza en el tratamiento de la. XXV Congreso de la Sociedad Española de Diabetes (SED). Pamplona: Sociedad Española de Diabetes (SED). $\begin{array}{lllllll}\text { Recuperado el } & 08 & \text { de } & 019, & \text { de }\end{array}$ https://www.diariodenavarra.es/noticias/navarra/mas_navarra/2014/04/05/el_entrenamien to_fuerza_mejora_tratamiento_obesidad_diabetes_154297_2061.html

Murillo, S. (01 de Noviembre de 2011). Fundacion para la diabetes. Recuperado el 10 de 08 de 2019, de Fundacion para la diabetes: https://www.fundaciondiabetes.org/general/articulo/57/prevencion-de-la-diabetes-tipo-2mediante-alimentacion-y-ejercicio

Murillo, S. (27 de abril de 2015). Fundación para la Diabetes. Recuperado el 06 de 08 de 2019 , de fundaciondiabetes.org: https://www.fundaciondiabetes.org/general/articulo/125/laimportancia-del-ejercicio-fisico-en-personas-con-diabetes

Sesmilo, G., \& Pérez, L. (28 de 06 de 2017). Endocs. Endocrinología y Nutrición. Recuperado el 06 de 08 de 2019, de endocrino.cat/es: http://www.endocrino.cat/es/blogendocrinologia.cfm/ID/8290/ESP/la-importancia-ejercicio-fisico-diabetes-tipo-2.htm

Suárez, L. (02 de 05 de 2019). Hospital Sant Joan De Déu Barcelona. Recuperado el 11 de 08 de 2019 , de sjdhospitalbarcelona.org/es: https://diabetes.sjdhospitalbarcelona.org/es/diabetes-tipo-1/consejos/pueden-realizarcualquier-tipo-ejercicio-ninos-diabetes 